\title{
Optimisation of a BCI system using the GA tehnique
}

\author{
Dan-Marius Dobrea \\ Faculty of Electronics, Telecommunications and \\ Information Technology \\ Technical University „Gheorghe Asachi““ \\ Iasi, Romania \\ mdobrea@etti.tuiasi.ro
}

\author{
Monica-Claudia Dobrea \\ Faculty of Electronics, Telecommunications and \\ Information Technology \\ Technical University „Gheorghe Asachi“ \\ Iasi, Romania \\ serbanm@etti.tuiasi.ro
}

\begin{abstract}
This paper, that continues a previous research, has as primer goal the improvement of a brain computer interface (BCI) system that uses a new features extracting method named Adaptive Nonlinear Amplitude and Phase Process (ANAPP). The ANAPP method models the EEG signals as a combination of five a priori "spontaneous cortical oscillations" whose amplitudes and phases are established using an adaptive algorithm. While in a series of previous researches [1], [2] the amplitude features of the model were extensively used, in this research the opportunity of using supplementary the phase information within the BCI system is analyzed. In addition, in this paper, the number and the type of the input features that feed the classification system are optimized using a GA algorithm. The final goals are to obtain both a faster BCI system and better classification results.
\end{abstract}

Keywords-brain computer interface; genetic algorithm; neural network; optimisation; mental task; Adaptive Nonlinear Amplitude and Phase Process

\section{INTRODUCTION}

An EEG-based brain computer interface (BCI) system is a device able to acquire, process, interpret and command other devices or systems based on the electroencephalographic (EEG) signals. Usually, the classification subsystem, an integrant part of any BCI application, uses one or more features extracted from the EEG signals in order to discriminate different mental tasks reflected within the EEG signals dynamics.

The EEG features, used for mental tasks classification and reported in the literature, are of rare encountered diversity. The most frequently used features in the BCI systems are: AR coefficients [3], [4], [5], [6], AR models with exogenous inputs [6], power spectral parameters [8], [7], [9], [10], [11], statistic phase synchronization [8], [9], spatial filtering [12], mean value of the phase coherence [8], discharge frequency of a neuronal group [15], P300 wave [12], [13], [14] etc.

This paper focuses on the same issue, namely that of finding more appropriate EEG features for cognitive tasks applications. The results presented in this paper are part of a more complex project whose final goal is to design and implement an autonomic self-organizing robotic system, mentally commanded by an user that gives one of the following four associated commands: forth, back, left, right. As the system is intended to be an on-line one, in the research conducted and reported in this paper the optimization of a previous developed BCI system [1], [2] is taken into consideration. The BCI system presented in [1], [2] uses in the

This work was supported entirely by the Romanian National University Research Council under Grant ID 1552.
EEG processing stage a new method in order to extract the feature vectors; this approach was named Adaptive Nonlinear Amplitude and Phase Process (ANAPP) method.

The ANAPP method models the EEG signals based on a combination of five a priori "spontaneous cortical oscillations" whose amplitudes and phases are established by an adaptive algorithm. These spontaneous cortical oscillations, needed within the ANAPP modeling, were previously derived using the coherence function calculated for each specific task. The resulting parameters obtained from the ANAPP model were then used as input data for a multi layer perceptron (MLP) artificial neural network (ANN) who finally provided the corresponding correct classification class.

Up to now, in the previous researches [1], [2], we have used only the amplitude features as inputs for the ANN in order to obtain improved classification performances. Unlike these, the present study has two main objectives. The first objective is to determine if the phase features, alone or together with the amplitude features, can be used to improve the classification performances. The second objective is to optimize the number of input features (amplitude, phase or both of them), using for this a genetic algorithm (GA), in order to obtain: superior classification performances, higher ANN generalization characteristics and a fast BCI system.

\section{MATERIALS AND METHODS}

\section{A. Data Acquisition}

The EEG data used in this paper was acquired from four subjects during performing five different mental tasks. The EEG signals were recorded from 3 pairs of electrodes placed on the scalp on the: central $(\mathrm{C} 3, \mathrm{C} 4)$, parietal $(\mathrm{P} 3, \mathrm{P} 4)$ and occipital $(\mathrm{O} 1, \mathrm{O} 2)$ positions, respectively. All EEG channels were referred to the right mastoid A2 and were sampled at 250 $\mathrm{Hz}$. Each recording time was $10 \mathrm{~s}$.

The subjects performed all the tasks without vocalizing and with the eyes closed. The tasks were as follows [2]: the baseline task (the subject relaxed as much as possible), the letter task (the subject mentally composed a letter to a friend), the counting task (the subject watched sequentially numbers written on an imaginary blackboard), the math task (the subject performed a nontrivial multiplication) and the rotation task (the subject studied for 30 seconds a 3D object and, with the object being removed, he was asked to imaginary rotate it 
around an axis). Moreover, the subjects performed two different trails for each task.

\section{B. The coherence function}

The coherence function indicates, in the frequency domain, the degree of linear correlation between two different signals. A zero value for the coherence function means the independence between the two signals while a value of 1 means the complete linear dependence.

The coherence estimate for each pair of signals, $x(t)$ and $y(t)$, was computed using the following formula:

$$
\left|\hat{R}_{x y}(\lambda)\right|^{2}=\frac{\left|\hat{f}_{x y}(\lambda)\right|^{2}}{\hat{f}_{x x}(\lambda) \hat{f_{y y}}(\lambda)} .
$$

The auto- and cross-spectra from the equation (1) were estimated by averaging over some overlapped sections:

$$
\hat{f}_{x y}(\lambda)=\frac{1}{2 \pi L T} \sum_{l=1}^{L} F_{x}^{T}(\lambda, l) \overline{F_{y}^{T}(\lambda, l)}
$$

In the relation (2) the over bar ' - , on $F^{T}{ }_{x}(\lambda, 1)$ indicates a complex conjugate and $(x, y)$ are the pairs $\left(s_{1}, s_{2}\right)$ for crossspectra, and $\left(s_{1}, s_{1}\right)$, respectively $\left(s_{2}, s_{2}\right)$ for autospectra; here, by $s_{1}$ and $s_{2}$ we denoted two different EEG signals, recorded from the same electrode position at different time moments. In order to obtain the formula for the auto- and cross-spectra function we first divided each of the two investigated time series into $L$ overlapping windows of length $T$. The $x(t)$ was replaced in our case by $s_{1}(t)$ - the first trial for each subject, each task and each EEG channel and respectively, by $s_{2}(t)$ - the second trial for the same subject, task and channel. To facilitate the consistence of the interpretation we calculated the pooled coherence estimate [2] based on a statistical test. The entirely methodology used to derive the a priori spontaneous cortical oscillations is presented in [2].

\section{Adaptive Nonlinear Amplitude and Phase Process}

After determining the spontaneous EEG frequencies based on the coherence function, an adaptive amplitude and phase model was implemented in order to model the original EEG time series.

The main hypothesis of the ANAPP model is constructed on the assumption that the EEG signal can be suitably be decomposed into several frequency components (some spontaneous oscillations - a priory specified) and their nonlinearly coupled frequencies (self-coupling oscillations and, respectively, cross-coupling oscillations). More exactly, two oscillatory waves (of $f_{1}$ and $f_{2}$ frequency - e.g., signals generated by two cortical oscillators), passing through a nonlinear square system (different neuronal pathways) generate two kinds of harmonic frequencies: self-coupling harmonics $\left(2 \cdot f_{1}\right.$ and $\left.2 \cdot f_{2}\right)$ and cross-coupling harmonics $\left(f_{1} \pm\right.$ $\left.f_{2}\right)$, respectively.

Correspondingly, the modeled EEG signal, $y[n]$, was assumed to be composed of $K$ (in our case $K=5$ ) different oscillations $\left(x_{j}, j=1 \div K\right)$. In the equations presented in (3) $T_{S}$ is the sampling rate, $f_{j}$ is the dominant $j$-th frequency, $\phi_{j}$ is its corresponding initial phase and $a, b, c$ and $d$ are the amplitude parameters of the model. The amplitude and phase parameters of the EEG model were then used as input features for the classification system.

$$
\begin{aligned}
& \left\{\begin{array}{l}
y[n]=\sum_{m=1}^{K} a_{m}[n] x_{m}[n]+\sum_{m=1}^{L} b_{m}[n] \alpha_{m}[n]+ \\
+\sum_{l=1}^{M} \sum_{\substack{m=1 \\
l \neq m}}^{N} c_{l m}[n] \beta_{l m}[n]+\sum_{l=1}^{P} \sum_{\substack{m=1 \\
l \neq m}}^{Q} d_{l m}[n] \theta_{l m}[n]
\end{array}\right. \\
& x_{j}[n]=\sin \left(n \cdot T_{S} \cdot 2 \pi \cdot f_{j}+\phi_{j}\right) \\
& \alpha_{i}[n]=\sin (n \cdot T_{S} \cdot 2 \pi \cdot \underbrace{2 f_{j}}_{f_{i}}+\varphi_{i}) \\
& \beta_{i j}[n]=\sin \left[n \cdot T_{S} \cdot 2 \pi \cdot\left(f_{i}-f_{j}\right)+\phi_{i j}\right] \\
& \theta_{i j}[n]=\sin \left[n \cdot T_{S} \cdot 2 \pi \cdot\left(f_{i}+f_{j}\right)+\varphi_{i j}\right]
\end{aligned}
$$

The least mean square (LMS) algorithm was used in order to adaptively estimate the model parameters (i.e., the amplitudes $a_{j}, b_{j}, c_{i j}, d_{i j}$ and the phases $\phi_{\mathrm{i}}, \phi_{i j}, i, j=1 \div K, i \neq$ $j)$. For this, the square error, given by:

$$
e[n]^{2}=[s[n]-y[n]]^{2}
$$

was used in the definition of the cost function $J$ (i.e., $J=$ $\left.1 / 2 \cdot E\left\{e[n]^{2}\right\}\right)$ that should be minimized. In equation (4), $s[n]$ is the real EEG signal while $y[n]$ is its corresponding modeled signal.

Applying the LMS relation, in [1], [2] we have obtained the following adjusting formulas for the ANAPP amplitude model parameters:

$$
\begin{gathered}
a_{j}[n+1]=a_{j}[n]+\eta_{a_{j}} e[n] x_{j}[n] \\
b_{j}[n+1]=b_{j}[n]+\eta_{b_{j}} e[n] \alpha_{j}[n] \\
c_{i j}[n+1]=c_{i j}[n]+\eta_{c_{i j}} e[n] \beta_{i j}[n] \\
d_{i j}[n+1]=d_{i j}[n]+\eta_{d_{i j}} e[n] \theta_{i j}[n]
\end{gathered}
$$

Regarding the relating phase parameters, after we applied Windrow's LMS relations, we obtained further: 


$$
\begin{gathered}
\phi_{j}[n+1]=\phi_{j}[n]+\eta_{\phi_{j}} \cdot e[n] \cdot a_{j}[n] \cdot \cos \left(n \cdot T_{s} \cdot 2 \pi \cdot f_{j}+\phi_{j}\right) \\
\varphi_{i}[n+1]=\varphi_{i}[n]+\eta_{\theta_{i}} \cdot e[n] \cdot b_{i}[n] \cdot \cos (n \cdot T_{S} \cdot 2 \pi \cdot \underbrace{2 f_{j}}_{f_{i}}+\varphi_{i}) \\
\phi_{i j}[n+1]=\phi_{i j}[n]+\eta_{\phi_{j}} \cdot e[n] \cdot c_{i j}[n] \cdot \cos \left(n \cdot T_{s} \cdot 2 \pi \cdot\left(f_{i}-f_{j}\right)+\phi_{i j}\right) \\
\varphi_{i j}[n+1]=\varphi_{i j}[n]+\eta_{\phi_{i j}} \cdot e[n] \cdot d_{i j}[n] \cdot \cos \left(n \cdot T_{s} \cdot 2 \pi \cdot\left(f_{i}-f_{j}\right)+\varphi_{i j}\right)
\end{gathered}
$$

\section{RESULTS AND DISCUSSIONS}

In order to compare the obtained results, presented in this paper, we focused only on one single subject, namely subject 2 , as in the papers [1] and [2]. The subject 2 was reported in the literature as having the greatest classification performances in comparison with the other 3 subjects.

The feature vectors employed at the input of the ANN classifier were obtained by concatenating the parameters of the ANAPP model calculated for EEG sliding windows of 256 samples recorded simultaneously from the all six EEG channels. The dimension of the feature vectors varied as a function of the analysis we made (i.e., only the amplitude coefficients were used, the phase coefficients or both coefficients types). All EEG channels were digitally high pass filtered with a FIR filter, having the cut off frequency at 20 $\mathrm{Hz}$.

For a sliding window of 2250 samples, overlapped by 12 samples, we finally got 1670 ANN input feature vectors (167 vectors per each recording $* 2$ recordings $* 5$ mental tasks). From this input database we have used $80 \%$ of data for the training set (1336 vectors) and $20 \%$ of data for the cross validation $(\mathrm{CV})$ set (334 vectors).

The necessity of digital pre-filtering the EEG signals issued from the largely accepted idea that the most important frequency peaks (the most part of the EEG signal power) are usually situated within the $0-20 \mathrm{~Hz}$ band, thus making us difficult to obtain a reliable ANAPP model for the EEG signal (almost all the a priori spontaneous cortical oscillations are situated at the frequency components superior to $20 \mathrm{~Hz}$ ). Additionally, in order that the proposed ANAPP model does not introduce frequencies within $0-20 \mathrm{~Hz}$ band or over the half of the sampling frequency (Shannon frequency, namely $125 \mathrm{~Hz}$ ), we have forced all the learning rates and the initial amplitude values to become zero but only for those derived frequencies within the already mentioned values intervals.

In this way, by removing the frequency components of

TABLE 1 THE CONFUSION MATRIX FOR THE 4 TASKS CLASSIFICATION CASE [1]

\begin{tabular}{|c|c|c|c|c|c|}
\cline { 3 - 6 } \multicolumn{2}{c|}{} & \multicolumn{4}{c|}{ Assigned classes } \\
\cline { 3 - 6 } \multicolumn{2}{c|}{} & $\mathrm{T} 2$ & $\mathrm{~T} 3$ & $\mathrm{~T} 4$ & $\mathrm{~T} 5$ \\
\hline \multirow{3}{*}{$\begin{array}{c}\text { Real } \\
\text { classes }\end{array}$} & $\mathrm{T} 2$ & $\mathbf{7 9 . 6 \%}$ & $3.2 \%$ & $6.3 \%$ & $10.9 \%$ \\
\cline { 2 - 6 } & $\mathrm{T} 3$ & $2.9 \%$ & $\mathbf{8 4 . 1} \%$ & $5.7 \%$ & $7.3 \%$ \\
\cline { 2 - 6 } & $\mathrm{T} 4$ & $6.1 \%$ & $13.6 \%$ & $\mathbf{7 7 . 3} \%$ & $3 \%$ \\
\cline { 2 - 6 } & $\mathrm{T} 5$ & $14.7 \%$ & $10.3 \%$ & $2.9 \%$ & $\mathbf{7 2 . 1 \%}$ \\
\hline
\end{tabular}

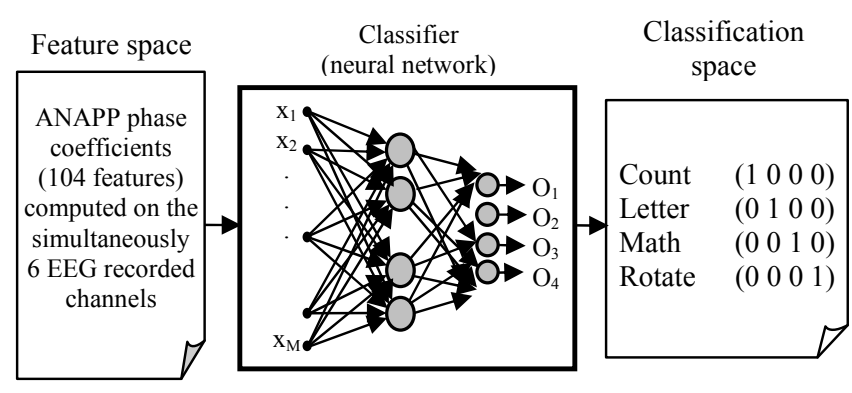

Figure 1. The classification process

zero value, the amplitude feature vectors were reduced from 180 components to 104 components. The same situation occurred in the case of phase feature set.

Further on, using the ANAPP model of the EEG signals, a MLP classifier and the methodologies presented in the papers [1] and [2], we obtained the results presented in Table 1. In order to obtain these results the ANN topology, the learning rates and the moment rates were extensively investigated. This searching work for the best ANN configuration was done based on a human expert strategy and a large number of trails having as main objective to obtain the best classification performance. The optimal architecture for the MLP ANN based on which we have obtained the best performances consisted in one input layer with 104 inputs, one hidden layer with 40 processing elements (PEs) and 4 outputs neurons. Each of these output neurons corresponded to the 4 mental tasks used in the classification process.

The results presented in Table 1 will be considered, in the rest of this paper, as a reference; moreover, having in mind the main final goal - that of obtaining a faster system and, finally, a real time system - in all analyses and discussions that will follow, we will try to improve these performances. In the same time, the ANN, based on which we obtained these first results, will be considered also as a reference neuronal network.

\section{A. The EEG phase features}

In a first analysis we wished to test the abilities of the phase features only to confine useful information able to obtain higher correct classification rates. For this analysis the input and the desired data sets were constructed as it is shown in Fig. 1.

The results obtained for the above presented case are given in Table 2. In this table, as in the all following tables, the tasks are represented as follows: T2 - count, T3 - letter, T4 - math, and $\mathrm{T} 5$ - rotate.

TABLE 2. THE CONFUSION MATRIX FOR THE 4 TASKS CLASSIFICATION CASE USING ONLY THE PHASE FEATURES

\begin{tabular}{|c|c|c|c|c|c|}
\cline { 3 - 6 } \multicolumn{2}{c|}{} & \multicolumn{4}{c|}{ Assigned classes } \\
\cline { 3 - 6 } \multicolumn{2}{c|}{} & $\mathrm{T} 2$ & $\mathrm{~T} 3$ & $\mathrm{~T} 4$ & $\mathrm{~T}$ \\
\hline \multirow{3}{*}{$\begin{array}{c}\text { Real } \\
\text { classes }\end{array}$} & $\mathrm{T} 2$ & $\mathbf{3 5 . 5 4 \%}$ & $\mathbf{1 7 . 5 2} \%$ & $31.35 \%$ & $15.59 \%$ \\
\cline { 2 - 6 } & $\mathrm{T} 3$ & $\mathbf{2 0 . 1 9 \%}$ & $\mathbf{5 1 . 2} \%$ & $16.27 \%$ & $12.34 \%$ \\
\cline { 2 - 6 } & $\mathrm{T} 4$ & $18.29 \%$ & $16.39 \%$ & $\mathbf{5 4 . 7 9 \%}$ & $10.53 \%$ \\
\cline { 2 - 6 } & $\mathrm{T} 5$ & $12.71 \%$ & $\mathbf{2 4 . 9 4 \%}$ & $15.75 \%$ & $\mathbf{4 6 . 6} \%$ \\
\hline
\end{tabular}


One can easily remark that using only the phase features of the ANAPP model the resulting classification performances are significantly inferior to those considered in this paper as reference (see Table 2 versus Table 1). In conclusion, the phase parameters of the ANAPP model are able to differentiate the four tasks, but the classification performances are inferior comparing with the ones based only on the amplitude parameters of the ANAPP model.

In the second analysis we tested the ability of both amplitude and phase features to obtain a synergic effect and, as a result, to get higher correct classification rates then those obtained using only the amplitude features or only the phase features. But, in spite of all expectations, the classification results proved to be even more inferior then those obtained in Table 2. An explanation for this behavior could be, among other possible explanations, a technical drawback, namely the size of the training set that is in this case quite small.

The required number of training feature vectors, $N$, necessary to classify correctly a test set with an error of $\varepsilon$ can be approximately given by the following relation [16]:

$$
N>\frac{W}{\varepsilon}
$$

In the previous relation $W$ is the number of weights in the ANN. In our particular case, considering an ANN with 208 inputs (104 amplitude features and 104 phase features), a number of at least 40 neurons on the hidden layer and 4 outputs neurons, a number of 8480 weights results. If an error of $10 \%$ is considered, the minimum number of the training feature vectors should then be greater than 84800 . But, we have only a training set of 1336 vectors. As a result, the size of the training data could be a reason for the lower classification performances obtained previously. Considering the above presented facts and the existing constrains (i.e., the size of the data set) a method able to select only those useful features (both phase and amplitude) capable to generate superior classification rates could be taken further in the consideration.

\section{B. GA optimization}

In order to solve the problem of the size of the feature vectors set a GA was used. The GA method selects from the entire feature set only those features (in our analysis these can be the amplitude features, the phase features only or both types of features), which are important for the classification process (i.e., those inputs that confine some special characteristics and information that assure the best discrimination characteristics between the data classes).

A chromosome used by the GA approach was composed from a series of values. In our case, only two values were allowed, namely 0 and 1 . The number of values ( 0 or 1 ) from a chromosome was set equal with the number of elements from the feature vector (also equal with the number of inputs for the MLP classifier). These inputs were then selected or de-selected using the genetic algorithm - a 0 value deselected the corresponding input while a value of 1 selected the corresponding input. The average cost (square average cost of the network's output) of the cross validation set was used as the

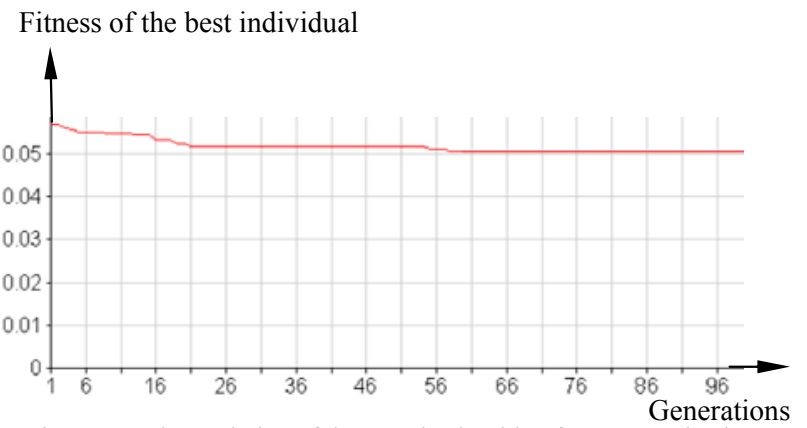

Figure 2. The evolution of the genetic algorithm for an ANN having as inputs the amplitude and the phase features

fitness criteria for the genetic algorithm. This cost should be minimized by the GA.

Usually, this optimization technique requires that the ANN be trained multiple times in order to find the optimal combination of inputs that produces the lowest error (e.g. a population of 50 chromosomes requires, in each generation, 50 ANN trainings). From this reason it becomes necessary to find - before starting the GA optimization -, an optimal MLP (topology, learning rates, moment rates, nonlinearity types, etc.) that has optimal convergence characteristics; that is, first, the networks have to have a stabile dynamics and, second, the ANN has to have a lower convergence time. In this mode, the time spent by the ANN is minimized and, as a result, each GA generation takes less time.

Using this approach (namely, the GA optimization of the feature vectors size), the previous classification problem we had when using both amplitude and phase features can be further over passed.

In Fig. 2 the evolution of the genetic algorithm displaying fitness of the best individual (the square average cost on the cross-validation set for the best chromosome of each generation) is presented. From this figure one can observe the ability of the GA algorithm to improve the classification performances using for this an optimal set of feature vectors continuously selected during the evolution process. The associated classification performances obtained at the end of the GA evolutions are presented in Table 3. Unfortunately, these performances continue to be inferior of the ones presented in Table 1.

Due to the worst classification performances obtained with the phase features, in a second step of this last analysis we decided to further optimize only the amplitude feature set (i.e., the number and the specific amplitude feature selected).

After an extensive search, we found that an ANN with a single hidden neuronal layer, with 23 processing elements on

TABLE 3. THE CONFUSION MATRIX FOR THE 4 TASKS CLASSIFICATION CASE AFTER THE GA SELECTED AMPLITUDE AND PHASE FEATURES

\begin{tabular}{|c|c|c|c|c|c|}
\cline { 3 - 6 } \multicolumn{2}{c|}{} & \multicolumn{4}{c|}{ Assigned classes } \\
\cline { 3 - 6 } \multicolumn{2}{c|}{} & $\mathrm{T} 2$ & $\mathrm{~T} 3$ & $\mathrm{~T} 4$ & $\mathrm{~T} 5$ \\
\hline \multirow{3}{*}{$\begin{array}{c}\text { Real } \\
\text { classes }\end{array}$} & $\mathrm{T} 2$ & $\mathbf{5 5 . 3 8 \%}$ & $12.31 \%$ & $21.54 \%$ & $10.77 \%$ \\
\cline { 2 - 6 } & $\mathrm{T} 3$ & $10 \%$ & $\mathbf{7 4 . 2 9} \%$ & $7.14 \%$ & $8.57 \%$ \\
\cline { 2 - 6 } & $\mathrm{T} 4$ & $17.19 \%$ & $9.37 \%$ & $\mathbf{7 1 . 8 8} \%$ & $1.56 \%$ \\
\cline { 2 - 6 } & $\mathrm{T} 5$ & $10.29 \%$ & $23.53 \%$ & $5.88 \%$ & $\mathbf{6 0 . 3} \%$ \\
\hline
\end{tabular}




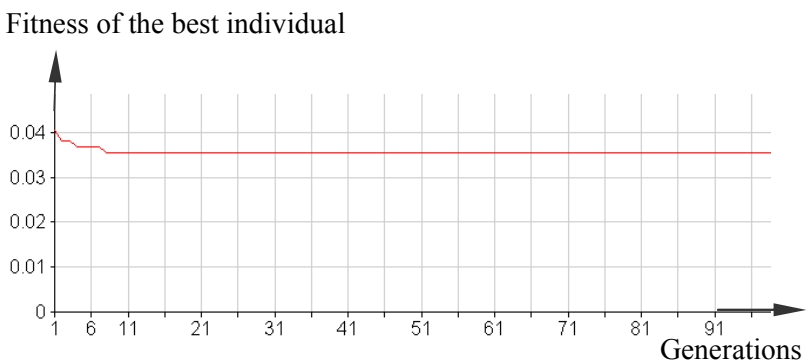

Figure 3. The evolution of the genetic algorithm

the hidden layer, having all moment rates equal to 0.95 , using a learning rate of 0.35 on the hidden layer and a learning rate of 0.03 on the output layer, had the best convergence characteristics for our specific case.

The GA was implemented based on a population of 15 chromosomes, using a roulette selection method, a uniform crossover operator and a classical mutation operator. The probabilities for crossover and mutation operators were set to 0.9 and 0.001 . Also, we tested different crossover methods (one point and two points), as well as different selection schemes: rank, tournament, stochastic uniform sampling, and stochastic remainder sampling. As a result, for our problem the most efficient crossover operator proved to be the uniform one, while the most effective selection scheme proved to be the roulette. A genetic evolution run until the maximum number of generations, one hundred in our case, was reached.

In Fig. 3 we present the evolution of the genetic algorithm displaying fitness of the best individual (the square average cost on the cross-validation set for the best chromosome of each generation).

The classification results obtained from the best chromosome, on the cross validation set, after the convergence of the GA are presented in Table 4.

If we compare the results presented in Table 4 with the reference classification rates, presented in Table 1, we observe only a slightly improvement of the classification performances obtained for the GA optimization techniques. The sum of the diagonal elements from Table 1 is 313.1 (the correct classification rates) and 315.05 for Table 4. Based on this information, the reference mean classification performance is 78.275; meanwhile the mean classification performance of all 4 tasks using the GA optimization techniques is 78.7625 .

The obtained improvement in the classification performances seem to be insignificant, and, consequently, inconsistent, but this slightly improvements is also sustained by a different paradigm. In this second paradigm an artificial neural network of only 23 processing elements on the hidden layer was used as compared to the 40 processing elements of

TABLE 4. THE CONFUSION MATRIX FOR THE 4 TASKS CLASSIFICATION CASE AFTER THE GA AMPLITUDE FEATURES OPTIMISATION

\begin{tabular}{|c|c|c|c|c|c|}
\cline { 3 - 6 } \multicolumn{2}{c|}{} & \multicolumn{4}{c|}{ Assigned classes } \\
\cline { 3 - 6 } \multicolumn{2}{c|}{} & $\mathrm{T} 2$ & $\mathrm{~T} 3$ & $\mathrm{~T} 4$ & $\mathrm{~T} 5$ \\
\hline \multirow{3}{*}{$\begin{array}{c}\text { Real } \\
\text { classes }\end{array}$} & $\mathrm{T} 2$ & $\mathbf{8 2 . 8 1 \%}$ & $6.25 \%$ & $4.68 \%$ & $6.26 \%$ \\
\cline { 2 - 6 } & $\mathrm{T} 3$ & $2.9 \%$ & $\mathbf{7 9 . 7 1 \%}$ & $7.25 \%$ & $10.14 \%$ \\
\cline { 2 - 6 } & $\mathrm{T} 4$ & $6.06 \%$ & $7.58 \%$ & $\mathbf{8 6 . 3 6 \%}$ & $0 \%$ \\
\cline { 2 - 6 } & $\mathrm{T} 5$ & $11.76 \%$ & $13.24 \%$ & $8.82 \%$ & $\mathbf{6 6 . 1 8} \%$ \\
\hline
\end{tabular}

the reference neural network. Another important difference comparing to the reference ANN, and which represents, in fact, the main advantage of this second used ANN, results directly from the implemented GA optimization process. Thus, instead of the 104 inputs of the reference ANN a number of only 80 selected inputs were used. As a direct result, the classification system's complexity decreased from a number of 4320 weights (104 inputs $\cdot 40$ neurons +40 neurons $\cdot 4$ neurons) for the reference ANN to only 1932 weights ( 80 inputs $\cdot 23$ neurons + 23 neurons $\cdot 4$ neurons) for the optimized ANN. Thus, the complexity of the new classification system was less then a half $(55.27 \%)$ of the reference ANN.

The system's complexity reduction has a number of advantages. First, the system is faster due to the decreasing number of computation associated with: each weight updating (during the backpropagation algorithm) and new class association of the input feature vector (in the forward step).

Second, using the same training data set, the classification performances, the generalization abilities of the new ANN, can be increased using a smaller number of amplitude feature vectors, see relation (13). From the relation (13) it can be observed the fact that having the same data set and a smaller set of weight (more then a half) the error can be decreased correspondingly and the classification rate can be increased.

\section{CONCLUSSIONS}

From the results presented previously one can remark the ability of the GA to optimize the feature data set in order to obtain both higher classification rates and a smaller complexity of the ANN. By using the GA, the complexity of the optimized ANN has been reduced to less then a half - with a number of 2388 weights. In this mode, we obtained a faster neural network. Using the GA optimization technique we made an important step to be closer to the final objective: a real time BCI system.

Regarding the phase parameters of the ANAPP model, one can conclude that this type of EEG features does not bring new information and discrimination power for the BCI system. Two possible explanations for this behavior could be taken into account in this case: first, in our analysis the instant value of the phase parameter was used instead of the derived form of it (which was suggested to care more information) and, second, the constrains imposed by the ANAPP model, namely, the selection of only five fundamental spectral components that removed probably some useful information.

This research emphasis once again the importance of the data training size and confirms the performances of the ANAPP model.

\section{ACKNOWLEDGMENT}

This work was entirely supported by the Romanian National University Research Council under Grant ID 1552.

\section{REFERENCES}

[1] D. M. Dobrea, and M. C. Dobrea, "EEG Classification System - From an Universal System Implementation to a Particular Signal Modeling," Proceedings of the Romanian Academy - Series A: Mathematics, Physics, Technical Sciences, Information Science, Vol. 10, Nr. 2, MayAugust 2009, pp. 197-204, ISSN 1454-8267 
[2] D. M. Dobrea, and M. C. Dobrea, "An EEG (Bio)Technological System For Assisting the Disabled People," Proceeding of the 5th International Conference on Computational Cybernetics, ICCC 2007, October 19-21 2007, Gammarth, Tunisia, pp. 191-196

[3] C. Guger, A. Schlögl, C. Neuper, D. Walterspacher, T. Strein, and G. Pfurtscheller, "Rapid prototyping of an EEG-based brain-computer interface (BCI)," IEEE Transactions on Neural Systems and Rehabilitation Engineering, vol. 9, nr. 1, 2001, pp. 49-58

[4] C. W. Anderson, E. Stolz, S. Shamsunder, "Multivariate Autoregressive Models for Classification of Spontaneous Electroencephalogram During Mental Tasks," IEEE Transactions on Biomedical Engineering, vol. 45, nr. 3, pp. 277-286, 1998

[5] V. A. Maiorescu, M. C. Dobrea (Şerban), and A.M. Lazar, "Classification of EEG Signals Represented by AR Models for Cognitive Tasks - a Neural Network Based Method," International Symposium on Signal Circuits and System, vol. 2, 10-11 iulie 2003, Iasi, Romania, pp. 441-444

[6] D. P. Burke, S. P. Kelly, de P. Chazal, R. B. Reilly, and C. Finucane, "A parametric feature extraction and classification strategy for braincompSuter interfacing," IEEE Transactions on Neural Systems and Rehabilitation Engineering, vol. 13, nr. 1, 2005, pp. 12-17

[7] B. J. Culpepper, and R. M. Keller, "Enabling Computer Decisions Based on EEG Input," IEEE Trans. on Neural Syst. and Rehab. Eng., vol. 11, no. 4, 2003, pp. 354-360

[8] E. Gysels, and P. Celka, "Phase synchronization for the recognition of mental tasks in a brain-computer interface," IEEE Transactions on Neural Systems and Rehabilitation Engineering, vol. 12, nr. 4, 2004, pp. $406-415$
[9] M. C. Dobrea (Şerban), and D.M. Dobrea, "Discrimination between cognitive tasks - a comparative study, "Proceedings of the International Symposium on Signals Circuits and Systems - ISSCS 2005, Iaşi, România, 14 - 15 iulie 2005, Vol. 2, pp. 805 - 808, ISBN 0-7803-9029-6

[10] S. P. Kelly, E. C. Lalor, C. Finucane, G. McDarby, and R. B. Reilly, "Visual Spatial Attention Control in an Independent Brain-Computer Interface," IEEE Transactions on Biomedical Engineering, lucrare acceptată spre publicare, 2005, pp. $1-9$

[11] T. Müller, T. Ball, R. Kristeva-Feige, T. Mergner, and J. Timmer, "Selecting Relevant Electrode Positions For Classifiation Tasks Based On The Electro-Encephalogram," Medical \& Biological Engineering \& Computing, 2000, vol. 38, pp. 62-67

[12] G. Schalk, D. J. McFarland, T. Hinterberger, N. Birbaumer, and J. R. Wolpaw, "BCI2000: a general-purpose brain-computer interface (BCI) system," IEEE Transactions on Biomedical Engineering, vol. 51, nr. 6, 2004, pp. 1034-1043

[13] J. R. Wolpawa, N. Birbaumerc, D. J. McFarlanda, G. Pfurtschellere, and T. M. Vaughan, "Brain-computer interfaces for communication and control," Clinical Neurophysiology, vol. 113, 2002, pp. 767-791

[14] H. Serby, E. Yom-Tov, and G. F. Inbar, "An improved P300-based brain-computer interface," IEEE Transactions on Neural Systems and Rehabilitation Engineering, vol. 13, nr. 1, 2005, pp. $89-98$

[15] S. G. Mason, and G. E. Birch, "A general framework for brain-computer interface design," IEEE Transactions on Neural Systems and Rehabilitation Engineering, vol. 11, nr. 1, 2003, pp. 70-85

[16] S. Haykin, "Artificial Neuronal Networks: A Comprehensive Foundation,” IEEE Press, New York, 1995 